\title{
Anatomic assessment of the left main bifurcation and dynamic bifurcation angles using computed tomography angiography
}

\author{
O. Beton ${ }^{1}$, H. Kaplanoglu², B. Hekimoglu², M.B. Yilmaz ${ }^{1}$ \\ ${ }^{1}$ Department of Cardiology, Heart Centre, University Hospital, Cumhuriyet University, Sivas, Turkey \\ ${ }^{2}$ Department of Radiology, Dıskapi Yildirim Beyazit Research and Training Hospital, Ankara, Turkey \\ [Received: 19 July 2016; Accepted: 20 August 2016]
}

Background: An understanding of the left main coronary artery (LMCA) anatomy is important for accurate diagnosis and therapy. We aimed to investigate LMCA anatomy via 128-multisliced coronary computed-tomography-angiography (CCTA) in patients with normal LMCA.

Materials and methods: A total of 201 CCTA studies were included in this study. Anatomical features of LMCA including cross-sectional areas of the LMCA ostial, LMCA distal, left anterior descending artery ( $L A D)$ ostial and left circumflex artery (LCX) ostial, and degree of tapering and LMCA bifurcation angles (BA) in the form of LMCA-LCX BA, LMCA-LAD BA, LAD-LCX BA at end-diastole and end-systole. Results: The mean age was $55 \pm 11 ; 55.7 \%$ of patients were males. Right coronary artery was dominant in 173 (86.1\%) patients. Mean LMCA length was $10.0 \pm 4.5 \mathrm{~mm}$. The mean values of LMCA ostial, LMCA distal, LAD ostial and LCX ostial areas were $18.2 \pm 5.1 \mathrm{~mm}^{2}, 13.2 \pm 4.0 \mathrm{~mm}^{2}, 9.0 \pm 3.2 \mathrm{~mm}^{2}$ and $7.6 \pm \pm 2.8 \mathrm{~mm}^{2}$, respectively. LMCA ostial-distal area, LMCA distal-LAD ostial area and LMCA distal-LCX ostial area ratios were $\geq 1.44-<1.69$ in 47 (23.4\%), 53 (26.4\%), 47 (23.4\%) patients, respectively, and were $\geq 1.69-<1.96$ in 19 (9.5\%), 24 (11.9\%), 40 (19.9\%) patients respectively. Systolic motion modifies LMCA BAs; systolic motion begets an increment of LMCA-LAD angle in $72.6 \%$ of patients and decrement of $\angle A D-L C X$ angle in $75.6 \%$ of patients. Patients with T-shaped $L A D$ - LCX BA was shown to have significantly longer $L M C A$, larger $L A D$ ostial area, larger $L C X$ ostial area and higher diastolic-to-systolic range (DSR) of LAD-LCX BA compared to patients with Y-shaped LAD-LCX BA.

Conclusions: $L M C A$ with T-shaped distal BA was found to have significantly longer LMCA, larger LAD ostial area, larger LCX ostial area and higher DSR of distal BA compared to patients with Y-shaped distal BA. These findings may provide useful information for LMCA bifurcation stenting or designing dedicated stents for LMCA. (Folia Morphol 2017; 76, 2: 197-207)

Key words: coronary computed tomography angiography, left main coronary artery, anatomic dimensions and bifurcations angles

Address for correspondence: O. Beton, MD, Department of Cardiology, Heart Centre, University Hospital, Cumhuriyet University Postal Code: 58140, Sivas, Turkey. tel: +90 346 2581833, fax: +90 346 2191268, e-mail: obeton@cumhuriyet.edu.tr 


\section{INTRODUCTION}

Significant left main coronary artery (LMCA) disease is identified in $4-6 \%$ of patients undergoing coronary angiography and $24 \%$ of patients with acute coronary syndromes [25]. Revascularisation by coronary artery by-pass grafting (CABG) has been considered as the standard therapy for significant LMCA disease by improving long-term survival when compared to optimal medical therapy [3]. Advances in the drug eluting stent (DES) have made the percutaneous coronary intervention $(\mathrm{PCl})$ more acceptable therapy as an alternative to CABG [3]. Randomised trials demonstrated comparable efficacy and safety between $\mathrm{PCl}$ and $C A B G$ for significant LMCA disease [3, 4, 26, 31].

Distal LMCA stenosis can be presented with a wide variety of complexity [20, 26, 29]. PCI for distal LMCA stenosis is associated with relatively poor clinical outcomes [22, 29]. Angiographic in-stent restenosis which leads to repeat revascularisation is the main reason of distal LMCA PCI-related poor clinical outcomes [6]. Besides clinical predictors, anatomical factors are taken into account while making decision for type of revascularisation [31]. Presence or absence of bifurcation lesion, bifurcation type, distal bifurcation angle (BA) and characteristics of stenosis are generally used for anatomical score calculation in LMCA lesions [4, 31]. In the SYNTAX score, presence of LMCA distal BA $<70^{\circ}$ is an adverse lesion characteristics due to the anticipated difficulty in covering the ostium of the side branch when a stent become necessary after main branch stenting [28]. Additional anatomical predictors for cardiovascular outcomes were also identified: diastolic to systolic change of LMCA distal BA by using three-dimensional (3D) quantitative angiography and post-stenting minimal lumen area (MLA) by using intravascular ultrasound [11, 17].

Several studies have evaluated the LMCA BAs during the cardiac cycle in patients with normal LMCA $[18,24]$. We aimed to investigate the dynamic BAs and LMCA dimensions by using coronary computed tomography angiography (CCTA) in patients with normal LMCA.

\section{MATERIALS AND METHODS}

\section{Study population}

Two hundred and one CCTA examinations of 201 patients were carefully selected from among 640 consecutive CCTA examinations performed patients in Department of Radiology, Diskapi Yildirim Beyazit Research and Training Hospital between January 2015 and May 2015. The following criteria were applied for inclusion: morphologically normal LMCA and ostial to proximal parts of left anterior descending artery (LAD) and left circumflex artery (LCX) (absence of atherosclerotic changes and luminal reduction), not more than $40 \%$ stenosis in right coronary artery (RCA) and the remaining parts of LAD and LCX (mid to distal parts of LAD and LCX) on CCTA, absence coronary anomalies (including absence of LMCA) on CCTA, sinus rhythm, absence of structural (valvular, myocardial, congenital) abnormality on echocardiography and high-quality CCTA examination (no obscured coronary artery patterns due to identifiable artefacts). Demographic and clinical data of patients were obtained from medical records and hospital database. All patients gave informed consent for CCTA procedure. The study was approved by the Ethics Committee of the Diskapi Yildirim Beyazit Research and Training Hospital (reference number: 26.10.2015-26/12) and conducted in accordance with the Helsinki Declaration.

\section{CCTA imaging protocol}

Patients received a beta-blocker $90-120 \mathrm{~min}$ before the examination (25-50-100-150-200 mg of metoprolol orally, based on resting heart rate), if resting heart rate exceeded $65 \mathrm{bpm}$. All patients included in the study were in sinus rhythm. The heart rate of all patients was below 65 bpm with or without premedication during CCTA imaging. Nitroglycerin was not administered before scanning. CCTA was performed using a 128-row multidetector computed tomography scanner (GE Optima 660 SE 64 Detector 128-slice CT, GE Healthcare, Milwaukee, USA). The following scanning parameters were applied: detector collimation, $64 \times 0.625 \mathrm{~mm}$; kVp, 120; mAs, 400-500; pitch range, $0.2-0.26$; gantry rotation time, $0.35 \mathrm{~s}$; and slice thickness, $0.6 \mathrm{~mm}$. 'Standard' reconstruction filter was used. Electrocardiography (ECG) modulation was used in all CCTA examinations. First, a non-enhanced low-dose CT scan of the entire chest was performed. In the next step, a bolus of $80 \mathrm{~mL}$ nonionic contrast medium (iomeprol or iopromide) was intravenously injected (rate, $4 \mathrm{~mL} / \mathrm{s}$ ), via an 18-gauge catheter placed in the antecubital vein, followed by a bolus of $40 \mathrm{~mL}$ of saline. Scan delay was determined according to the Smart Prep programme (automatic bolus test; the region of interest was placed on the ascending aorta). The participants were instructed to maintain an inspiratory breath hold, during which the CT data and ECG trace were acquired. 


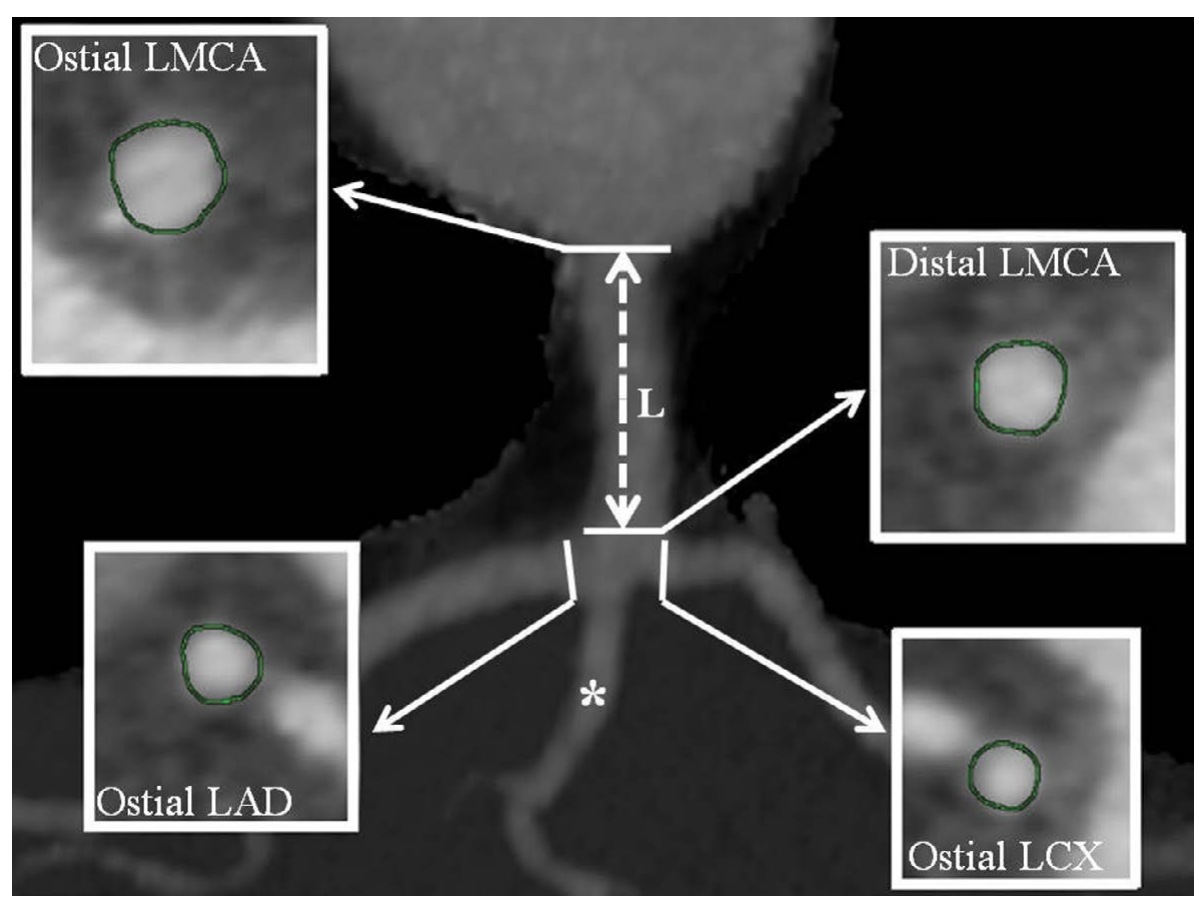

Figure 1. Cross-sectional areas of the three coronary arteries at the four points of each left main coronary artery (LMCA): ostial LMCA, distal LMCA at bifurcation level, ostial left anterior descending artery (LAD) at bifurcation level and ostial circumflex artery (LCX) at bifurcation level. The length of the LMCA was measured as the distance between ostial and distal LMCA (double head arrow). Artery came off exactly at the point of bifurcation it was labelled as ramus intermedius (asterisk); L — length of LMCA.

\section{Image reconstruction and data analyses}

Image reconstruction was done using the retrospective ECG gating method. Data sets were acquired at phases $40 \%, 70 \%$ and $80 \%$ of the R-R cycle. The image data sets were processed on a separate workstation (Advantage Workstation version 4.6, GE Healthcare, Milwaukee, USA), and analysed, using curved multiplanar reconstructions in various planes and thin-slab maximum-intensity projection reconstructions, in addition to the axial source images. Coronary artery findings were reviewed in consensus by one experienced radiologists and a cardiologist.

Cross-sectional reconstruction images were used to determine area of the LMCA, LAD and LCX. The plane of measurement was truly perpendicular to the long axis of the coronary arteries. Measurements were obtained during the end-diastolic phase at four points of each LMCA: ostial LMCA, distal LMCA at bifurcation level, ostial LAD at bifurcation level (LAD carina) and ostial LCX at bifurcation level (LCX carina) (Fig. 1). The length of the LMCA (distance between ostial and distal LMCA) was measured in all participants (Fig. 1). If an artery came off exactly at the point of bifurcation, it was labelled as ramus intermedius (intermediate artery), and this branching pattern was considered as trifurcation (Fig. 1). For the assessment of the aortocoronary junction (corresponding to the junction between the aorta and LMCA) 2D reconstructions were used. The cranial take-off angle of the LMCA at the aortocoronary junction was measured on coronal left oblique (between $30^{\circ}$ and $45^{\circ}$ ) plane view where segment of LMCA at least $1 \mathrm{~cm}$ in length were obtained (Fig. 2). 3D-volume rendering (VR) image was used for the assessment of LMCA BAs as follows: LMCA-LAD, LMCA-LCX and LAD-LCX (LMCA distal BA) (Fig. 3). $B A$ is measured via calculating the angle between central vectors of both arteries. First central vector is placed upon minimum $1 \mathrm{~cm}$ in length distal part of first vessel. Second central vector is placed upon minimum $1 \mathrm{~cm}$ in length distal part of second vessel. After that, junction of the two central vectors is taken as a centre of angle (this measurement is made on 3D-VR images). Each BA was measured both during end-diastolic phase and end-systolic phase (Fig. 4). LMCA distal BA was considered as Y-shaped when LMCA distal BA was $<70^{\circ}$ at end-diastole, and T-shaped when distal BA was $\geq 70^{\circ}$ at end-diastole. The term of "diastolic to systolic range (DSR)" was used to express absolute angle change regardless of the direction between end-diastole and end-systole 


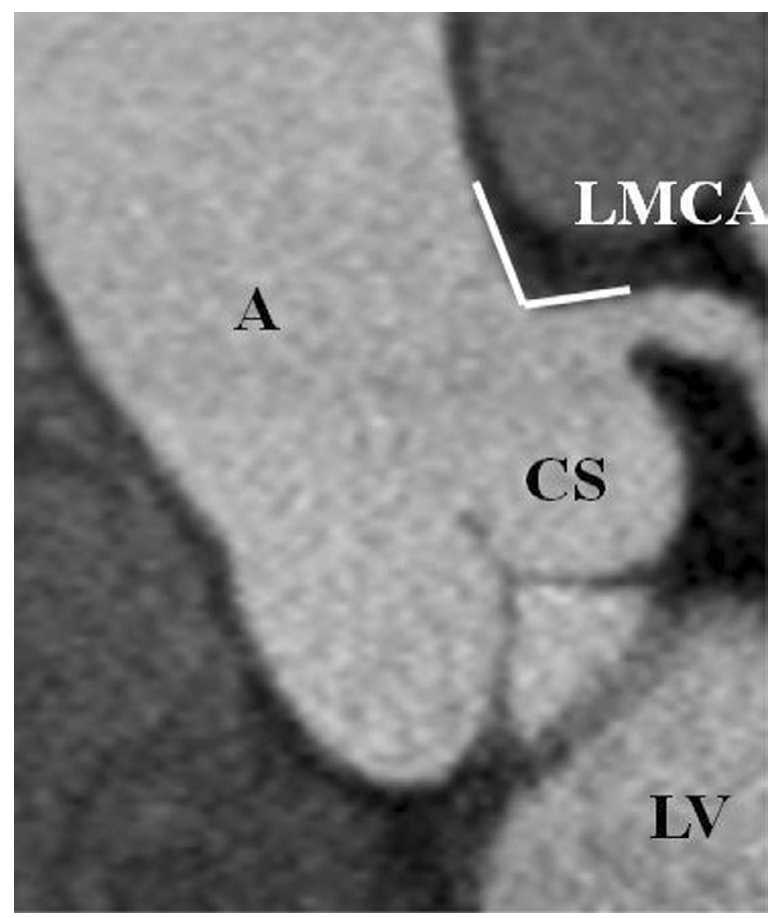

Figure 2. The cranial take-off angle of the left main coronary artery (LMCA) at the aortocoronary junction was measured on coronal left oblique plane; A — aorta; CS — left coronary sinus of Valsalva; LV - left ventricle.

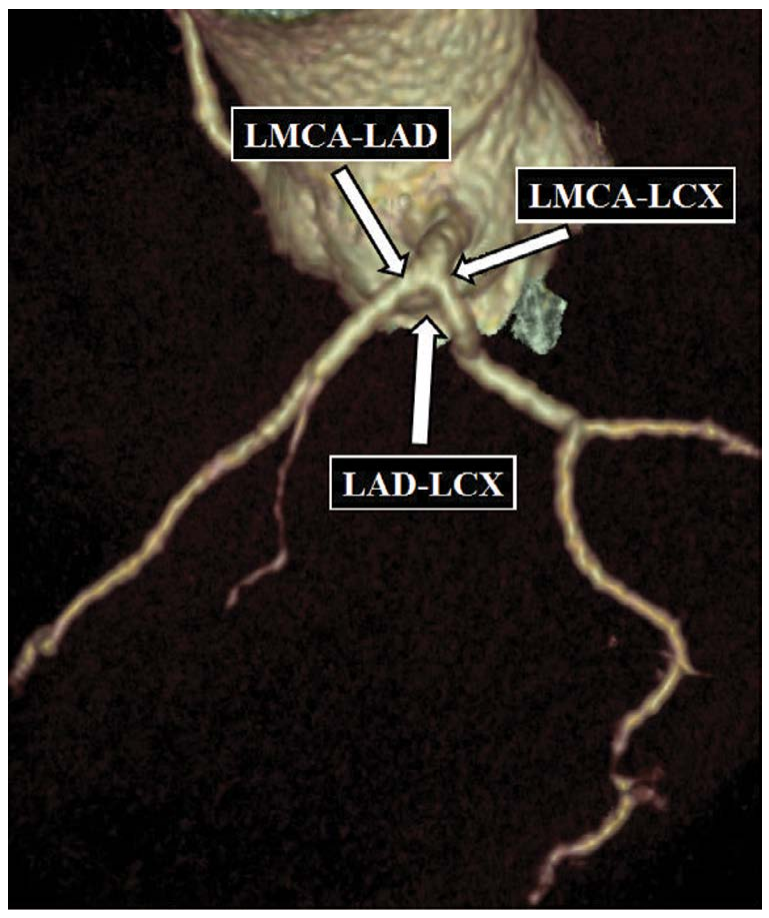

Figure 3. Three-dimensional volumetric reconstruction view of the angles at left main coronary artery (LMCA) bifurcation. LMCA-left anterior descending (LAD) artery angle, LMCA-left circumflex (LCX) artery angle, and LAD-LCX angle.
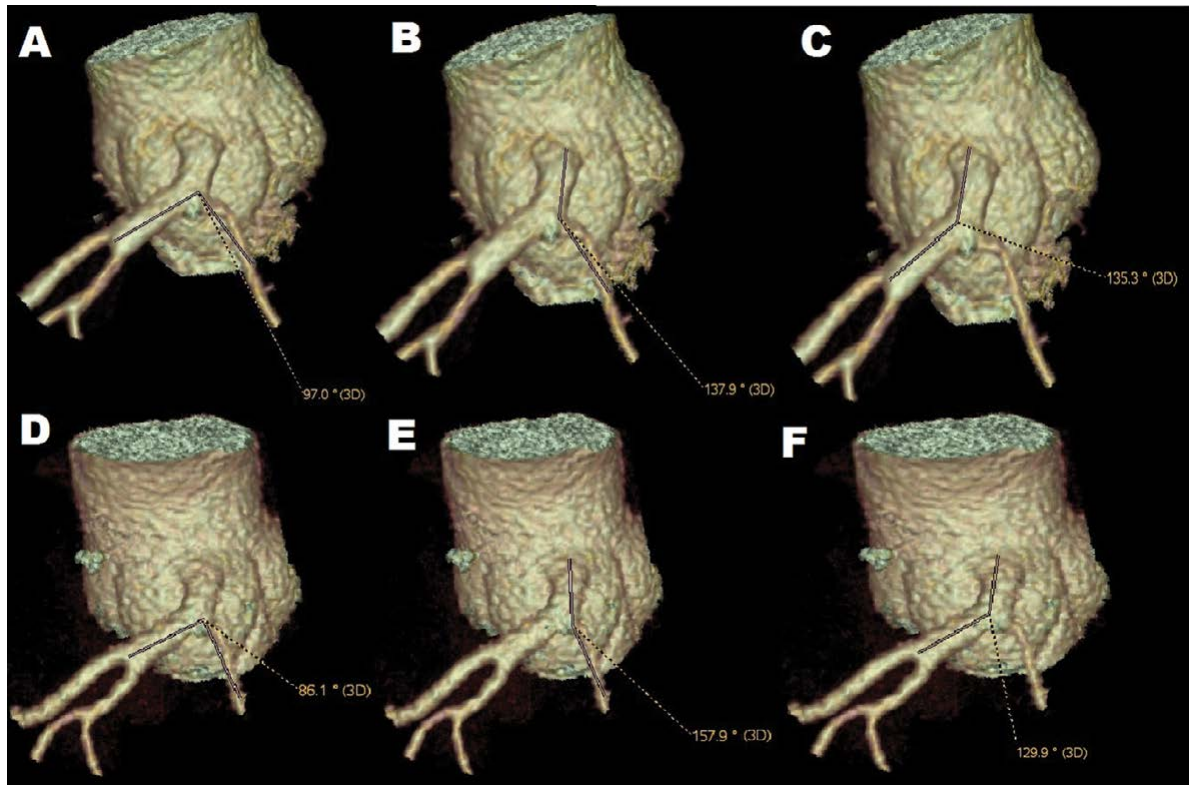

Figure 4. Left main coronary artery (LMCA) bifurcation angles of the representative case during end-diastolic phase (A-C) and end-systolic phase (D-F). Each bifurcation angles can be measured correctly with reproducibility on a volume rendering image using a three-dimensional workstation; A. End-diastolic angle of LAD-LCX; B. End-diastolic angle of LMCA-LCX; C. End-diastolic angle of LMCA-LAD; D. End-systolic angle of LAD-LCX; E. End-systolic angle of LMCA-LCX; F. End-systolic angle of LMCA-LAD; LAD — left anterior descending artery; LCX — left circumflex artery. 
and the term of "directional angular change (DAC)" was used to express angular change due to cardiac movement which was calculated as end-diastolic angle minus end-systolic angle.

Right coronary artery was considered dominant if the posterior descending artery (PDA) originated off the RCA, while LCX was considered dominant if the PDA originated off the LCX. Codominance was considered if both the LCX and the RCA contributed to the PDA. For the LMCA bifurcating trunks, three types were identified. Type I bifurcation was defined as the cross-sectional area of LAD greater than the cross-sectional area of $L C X(L A D>L C X)$. Type II bifurcation was defined as the cross-sectional area of $L A D$ equal to that of $L C X(L A D=L C X)$. Finally, a type III bifurcation was defined as the cross-sectional area of LCX greater than the cross-sectional area of LAD (LCX > LAD).

Tapering segments of arteries were classified into four groups according to ratio of proximal and distal area of the segment. Area calculation formula was $A=1 / 4 . \Pi . D 2$, where $A$ is lumen area and $D$ is lumen diameter. According to this formula, $A_{\text {proximal }} / A_{\text {distal }}$ can be simplified to $D_{\text {proximal }}{ }^{2} / D_{\text {distal }}{ }^{2}$. So, if $D_{\text {proximal }}$ $/ D_{\text {distal }}$ ratio is $1.2 / 1, A_{\text {proximal }} / A_{\text {distal }}$ is calculated to be 1.44 (if $D_{\text {proximal }} / \mathrm{D}_{\text {distal }}$ ratio is $1.3 / 1$ and $1.4 / 1, A_{\text {proximal }}$ $/ A_{\text {distal }}$ is calculated to be 1.69 and 1.96 , respectively). Ratios of LMCA osteal-LMCA distal, LMCA distal-LAD ostial and LMCA distal-LCX ostial areas were classified according to these values: $<1.44$ (insignificant tapering), 1.44- $<1.69$ (moderate tapering), 1.69- $<1.96$ (pronounced tapering) and $>1.96$ (very pronounced tapering).

\section{Statistical analysis}

Statistical analyses were performed using SPSS version 16.0 (IBM SPSS Statistics, IBM Corporation, Chicago, IL, USA). The variables were investigated using analytical method (One Sample KolmogorovSmirnov test) to determine whether or not they were normally distributed. Categorical variables were expressed as percentages and continuous variables as mean \pm standard deviation in the presence of normal distribution. In the presence of non-normal distribution, continuous variables were expressed as median (minimum-maximum). Comparisons between groups of patients were made using a $\chi^{2}$ test for categorical variables, an independent samples t-test for normally distributed continuous variables, and the Mann-Whitney $U$ test when distribution was skewed. Paired samples $T$ test was used to compare the change in angles from end-diastolic to end-systolic if the values were normally distributed, and Wilcoxon test was used if the distribution was skewed. While investigating the associations between normally distributed variables, the correlation coefficients and their significance were calculated using the Pearson test. In case of non-normally distributed variables and/or ordinal variables, the correlation coefficients and their significance were calculated using the Spearman test. A p value of 0.05 was considered statistically significant.

\section{RESULTS}

\section{Study population}

Baseline characteristics of the 201 patients examined are presented in Table 1. The mean age was $55 \pm 11$ and $55.7 \%$ of patients were males. RCA was dominant in $173(86.1 \%)$ patients. The most frequent division of LMCA was bifurcation (67.7\%). Trifurcation type was identified in $64(31.8 \%)$ patients and quadrifurcation type in $1(0.05 \%)$ patient. The cranial take-off angle of the LMCA at the aortocoronary junction was $116 \pm 16^{\circ}$. The most frequent bifurcation type of LMCA was type I (67.7\%), followed by type III (20.9\%) and type II (11.4\%).

\section{Dimensional and angular analyses of LMCA and LMCA bifurcation}

Dimensional analyses of LMCA and LMCA bifurcation of study population are presented in Table 1 . Mean LMCA length was $10.0 \pm 4.5 \mathrm{~mm}$. The mean values of LMCA ostial, LMCA distal, LAD ostial and LCX ostial areas were $18.2 \pm 5.1 \mathrm{~mm}^{2}, 13.2 \pm 4.0 \mathrm{~mm}^{2}$, $9.0 \pm 3.2 \mathrm{~mm}^{2}$ and $7.6 \pm 2.8 \mathrm{~mm}^{2}$, respectively. LMCA distal area was $\leq 8.2 \mathrm{~mm}^{2}$ in $5(2.5 \%)$ patients, ostial LAD area was $\leq 6.3 \mathrm{~mm}^{2}$ in 32 (15.9\%) patients and ostial LCX area was $\leq 5.0 \mathrm{~mm}^{2}$ in $28(13.9 \%)$ patients. The mean values of LMCA ostial-LMCA distal area ratio, LMCA distal-LAD ostial area ratio and LMCA distal-LCX ostial area ratio were $1.4 \pm 0.3,1.5 \pm 0.4$ and $1.8 \pm 0.5$, respectively (Table 1 ).

Degree of LMCA and LMCA bifurcation tapering are presented in Table 2. The vessel segment between LMCA ostial and distal showed moderate tapering in 47 (23.4\%) patients, pronounced tapering in $19(9.5 \%)$ patients, and very pronounced tapering in $11(5.5 \%)$ patients. The vessel segment between LMCA distal and LAD ostial showed moderate tapering in $53(26.4 \%)$ patients, pronounced tapering in $24(11.9 \%)$ patients, and very pronounced tapering in $19(9.5 \%)$ patients. 
Table 1. Baseline and coronary computed tomography (CT) angiographic characteristics of study population

\begin{tabular}{|c|c|}
\hline Variables & All patients ( $\mathrm{n}=201$ ) \\
\hline \multicolumn{2}{|l|}{ Clinical variables } \\
\hline Age [years] & $55 \pm 11$ \\
\hline Male gender & $112(55.7 \%)$ \\
\hline Hypertension & $110(54.7 \%)$ \\
\hline Diabetes mellitus & $54(26.9 \%)$ \\
\hline Hyperlipidaemia & $113(56.2 \%)$ \\
\hline Current smoker & $63(31.3 \%)$ \\
\hline Body mass index & $29 \pm 4$ \\
\hline Body surface area & $1.9 \pm 0.2$ \\
\hline \multicolumn{2}{|l|}{ Coronary CT angiography variables } \\
\hline Cranial take-off angle [degree] & $116 \pm 16$ \\
\hline \multicolumn{2}{|l|}{ Frequency of dominance: } \\
\hline RCA dominance & $173(86.1 \%)$ \\
\hline CX dominance & $15(7.5 \%)$ \\
\hline Codominant & $13(6.5 \%)$ \\
\hline \multicolumn{2}{|l|}{ LMCA division types: } \\
\hline Bifurcation & $136(67.7 \%)$ \\
\hline Trifurcation type & $64(31.8 \%)$ \\
\hline Quadrifurcation type & $1(0.05 \%)$ \\
\hline \multicolumn{2}{|l|}{ LMCA bifurcation types*: } \\
\hline Type I & $136(67.7 \%)$ \\
\hline Type II & $23(11.4 \%)$ \\
\hline Type III & $42(20.9 \%)$ \\
\hline \multicolumn{2}{|l|}{ LMCA dimensions: } \\
\hline LMCA length [mm] & $10.0 \pm 4.5$ \\
\hline LMCA ostial area [mm²] & $18.2 \pm 5.1$ \\
\hline LMCA distal area [mm²] & $12.6(4.4-30.9)$ \\
\hline LMCA distal area $<8.2 \mathrm{~mm}^{2}$ & $5(2.5 \%)$ \\
\hline LAD ostial area [mm²] & $8.4(2.9-25.6)$ \\
\hline LAD ostial area $<6.3 \mathrm{~mm}^{2}$ & $32(15.9 \%)$ \\
\hline LCX ostial area [mm²] & $7.2(2.2-20.8)$ \\
\hline LCX ostial area $<5.0 \mathrm{~mm}^{2}$ & $28(13.9 \%)$ \\
\hline LMCA ostial-LMCA distal area ratio & $1.4 \pm 0.3$ \\
\hline LMCA distal-LAD ostial area ratio & $1.5 \pm 0.4$ \\
\hline LMCA distal-LCX ostial area ratio & $1.8 \pm 0.5$ \\
\hline
\end{tabular}

*LMCA bifurcation types according to LAD and LCX including all LMCA division types. LCX - left circumflex artery; LMCA — left main coronary artery; RCA — right coronary artery

Angular analyses of LMCA bifurcation are presented in Table 3. End-diastolic angles of LMCA-LAD, LMCA-LCX and LAD-LCX were $153 \pm 15^{\circ}, 133 \pm 23^{\circ}$ and $85 \pm 23^{\circ}$, respectively. The median value of DSR which is the absolute angle change regardless of the direction during cardiac cycle was $7.0^{\circ}$ for LMCA-LAD, $11.1^{\circ}$ for LMCA-LCX and $9.7^{\circ}$ for LAD-LCX. DAC of LMCA-LAD from end-diastole to end-systole was statistically insignificant ( $p=0.755)$, whereas it was statistically significant for LMCA-LCX and LAD-LCX ( $p<0.001$ and $p<0.001$, respectively). The median value of DAC (end-diastolic minus end-systolic) of LMCA-LAD, LMCA-LCX and LAD-LCX was $-0.7^{\circ},-8.7^{\circ}$ and $8.3^{\circ}$, respectively. LMCA-LAD, LMCA-LCX and LAD-LCX BAs showed systolic increase in 103 (51.2\%), $146(72.6 \%)$ and 49 (24.4\%) patients, respectively, and systolic decrease in 98 (48.8\%), 55 (27.4\%) and $152(75.6 \%)$ patients, respectively.

\section{Y-shaped vs. T-shaped distal LMCA bifurcation angle}

Fifty-two patients had Y-shaped LMCA distal BA (distal BA $<70^{\circ}$ ) and 149 (74.1\%) patients had T-shaped LMCA distal BA in the study group. Characteristics of study population according to LMCA distal BA shape are presented in Table 4. Length of LMCA was higher in T-shaped group as compared to $\mathrm{Y}$-shaped group $(10.8 \pm 4.5 \mathrm{~mm}$ vs. $7.9 \pm 3.7 \mathrm{~mm}, \mathrm{p}<0.001)$. LAD ostial and LCX ostial areas were statistically higher in T-shaped group as compared to Y-shaped group ( $p=0.001$ and $p=0.034$, respectively). As expected, end-diastolic and end-systolic angles of LMCA-LAD and LMCA-LCX were higher in Y-shaped group. The median value of DSR of LMCA-LCX and LAD-LCX BAs were higher in T-shaped group compared to Y-shaped group (12.1 vs. 8.3, $p=0.004$ and 12.8 vs. 5.2, $\mathrm{p}<0.001$, respectively). DSR of LAD-LCX BA was $\geq 10^{\circ}$ in $88(59.1 \%)$ patients of T-shaped group and $10(19.2 \%)$ patients of $Y$-shaped group $(p<0.001)$. Similarly, DAC of LMCA-LCX and LAD-LCX BAs were higher in T-shaped group compared to Y-shaped group ( $p=0.011$ and $p<0.001$, respectively).

In patients with normal LMCA, end-diastolic distal LMCA BA were correlated with end-systolic distal BA $(r=0.826, p<0.001)$, DAC of distal BA $(r=0.431$, $p<0.001)$, DSR of distal LMCABA $(r=0.473, p<0.001)$, end-diastolic LMCA-LAD BA $(r=-0.421, p<0.001)$, end-systolic LMCA-LAD BA $(r=-0.395, p<0.001)$, end-diastolic LMCA-LCX BA $(r=-0.773, p<0.001)$, end-systolic LMCA-LCX BA ( $r=-0.661$, $p<0.001)$, LMCA length $(r=0.465, p<0.001)$, cranial angle between aorta and LMCA $(r=0.151, p=0.032)$, LAD ostial area $(r=0.200, p=0.04)$ and LCX ostial area $(r=0.156, p=0.027$ ) (Table 5). 
Table 2. Classification of degree of left main coronary artery (LMCA) and LMCA bifurcation tapering

\begin{tabular}{lcccc}
\hline Vessel segments & \multicolumn{4}{c}{ Tapering degree } \\
\cline { 2 - 5 } & $\mathbf{2 1 . 4 4}$ & $\geq \mathbf{1 . 4 4 - < \mathbf { 1 . 6 9 }}$ & $\geq \mathbf{1 . 6 9 - < \mathbf { 1 . 9 6 }}$ & $\geq \mathbf{1 . 9 6}$ \\
\hline LMCA ostial-LMCA distal ratio & $124(61.7 \%)$ & $47(23.4 \%)$ & $19(9.5 \%)$ & $11(5.5 \%)$ \\
LMCA distal-LAD ostial ratio & $105(52.2 \%)$ & $53(26.4 \%)$ & $24(11.9 \%)$ & $19(9.5 \%)$ \\
LMCA distal-LCX ostial ratio & $47(23.4 \%)$ & $47(23.4 \%)$ & $40(19.9 \%)$ & $67(33.3 \%)$ \\
\hline
\end{tabular}

LCX — left circumflex artery; LAD — left anterior descending artery

Table 3. Angular analyses of left main coronary artery (LMCA) bifurcation in the study population

\begin{tabular}{lc}
\hline Variables & All patients $\mathbf{( n = 2 0 1 )}$ \\
\hline LMCA-LAD angle & \\
End-diastole [degree] & $153 \pm 15$ \\
End-systole [degree] & $153 \pm 14$ \\
LMCA-LCX angle & \\
End-diastole [degree] & $133 \pm 23$ \\
End-systole [degree] & $141 \pm 21$ \\
LAD-LCX angle & \\
End-diastole [degree] & $85 \pm 23$ \\
End-systole [degree] & $75 \pm 22$ \\
Diastolic-systolic range & \\
LMCA-LAD [degree] & $7.0(0.5-30.4)$ \\
LMCA-LCX [degree] & $11.1(0.1-45.8)$ \\
LAD-LCX [degree] & $9.7(0.1-42.0)$ \\
Directional angular change & \\
LMCA-LAD [degree] & $-0.7(-28.3-30.4)$ \\
Systolic increase in LMCA-LAD & $103(51.2 \%)$ \\
Systolic decrease in LMCA-LAD & $98(48.8 \%)$ \\
LMCA-LCX [degree] & $-8.7(-45.8-34.0)$ \\
Systolic increase in LMCA-LCX & $146(72.6 \%)$ \\
Systolic decrease in LMCA-LCX & $55(27.4 \%)$ \\
LAD-LCX [degree] & $8.3(-19.9-42.0)$ \\
Systolic increase in LAD-LCX & $49(24.4 \%)$ \\
Systolic decrease in LAD-LCX & $152(75.6 \%)$ \\
\hline$C X-I f$ cic \\
\end{tabular}

LCX — left circumflex artery; LAD — left anterior descending artery

\section{DISCUSSION}

The major findings of this study of 201 normal LMCA evaluated by CCTA imaging: 1) LMCA ostialLMCA distal area and LMCA distal-LAD ostial area ratios were $\geq 1.44$ in 77 (38.4\%) and $96(47.8 \%)$ patients respectively which implicates moderate to very pronounced tapering structure of LMCA and LMCA bifurcation; 2) Systolic motion modifies LMCA BAs; systolic motion begets an increment of LMCA-LAD angle in $72.6 \%$ of patients and decrement of LAD-LCX angle in $75.6 \%$ of the patients; 3) DSR of LAD-LCX angle was $\geq 10^{\circ}$ in $98(48.8 \%)$ patients and $<10^{\circ}$ in 103 (51.2\%) patients; 4) Patients with T-shaped distal BA was shown to have significantly longer LMCA, larger LAD ostial area, larger LCX ostial area and higher DSR of LAD-LCX angle compared to patients with Y-shaped distal BA; 5) Both LAD and LCX ostial areas showed weak positive correlation with LMCA distal BA, whereas DSR of LAD-LCX angle showed moderate positive correlation with LMCA distal BA.

Appropriate stent expansion is crucial to prevent DES stenosis and thrombosis $[10,17]$. It is reported that MLA predicting in-stent restenosis after LMCA bifurcation stenting is $<5.0 \mathrm{~mm}^{2}$ for ostial LCX, $<6.3 \mathrm{~mm}^{2}$ for ostial LAD, $<7.2 \mathrm{~mm}^{2}$ for LMCA bifurcation segment and $<8.2 \mathrm{~mm}^{2}$ for LMCA $[17,20]$. LCX with $\geq 2.5 \mathrm{~mm}$ diameter is usually considered a major side branch favouring a two-stent technique in case of extended lesion to proximal LCX $[20,23]$. Presence of small LCX with $<2.5 \mathrm{~mm}$ luminal diameter (or $<4.9 \mathrm{~mm}^{2}$ MLA according to area calculation formula) favours provisional one-stent approach rather than two-stent approach [23]. In the present study, we found that $86.1 \%$ of patients have LCX area $>4.9 \mathrm{~cm}^{2}$.

Vessel tapering of a stenotic vessel is an important issue for stenting [30]. Stents have generally tubular shape and are designed to be selected slightly larger in diameter than artery for proper expansion [30]. Stent size selection is generally determined based on a stent-to-artery ratio of 1.0:1 to 1.2:1 [15]. Nevertheless, particularly in left main [5] and likewise long and bifurcated lesions [20,29], vessel tapering can be an important problem, which may not be adequately solved by conventional tubular stents [30]. Thus, a large diameter mismatch at the distal site of the stent may lead to damage in the arterial wall, increased wall stress, endothelial dysfunction and, 
Table 4. Characteristics of study population according to distal bifurcation angle

\begin{tabular}{|c|c|c|c|}
\hline Variables & Y-shaped ( $n=52$ ) & T-shaped (n=149) & $\mathbf{P}$ \\
\hline Male gender & $31(59.6 \%)$ & $81(54.4 \%)$ & 0.511 \\
\hline Body mass index $\left[\mathrm{kg} / \mathrm{m}^{2}\right]$ & $29 \pm 4$ & $29 \pm 4$ & 0.346 \\
\hline Body surface area $\left[\mathrm{m}^{2}\right]$ & $1.9 \pm 0.2$ & $1.9 \pm 0.2$ & 0.888 \\
\hline Dominant RCA & $48(92.3 \%)$ & $125(83.9 \%)$ & 0.202 \\
\hline Dominant LCX & $3(5.8 \%)$ & $12(8.1 \%)$ & 0.764 \\
\hline Coronal angle between aorta and LMCA [degree] & $113 \pm 17$ & $117 \pm 15$ & 0.099 \\
\hline LMCA length [mm] & $7.9 \pm 3.7$ & $10.8 \pm 4.5$ & $<0.001$ \\
\hline LMCA ostial area [mm²] & $18.2 \pm 4.1$ & $18.2 \pm 5.4$ & 0.937 \\
\hline LMCA distal area $\left[\mathrm{mm}^{2}\right]$ & $12.4(7.9-21.8)$ & $12.6(4.4-30.9)$ & 0.712 \\
\hline LAD ostial area $\left[\mathrm{mm}^{2}\right]$ & $7.5(4.2-14.0)$ & $8.9(2.9-25.6)$ & 0.001 \\
\hline LCX ostial area [mm²] & $6.7(3.8-12.0)$ & $7.5(2.2-20.8)$ & 0.034 \\
\hline LMCA ostial-distal area ratio: & $1.4 \pm 0.3$ & $1.4 \pm 0.3$ & 0.357 \\
\hline$<1.44$ & $31(59.6 \%)$ & $93(62.4 \%)$ & 0.721 \\
\hline$\geq 1.44-<1.69$ & $12(23.1 \%)$ & $35(23.5 \%)$ & 1.0 \\
\hline$\geq 1.69$ & $9(17.3 \%)$ & $21(14.1 \%)$ & 0.738 \\
\hline \multicolumn{4}{|l|}{ LMCA bifurcation types*: } \\
\hline Type I & $31(59.6 \%)$ & $105(70.5 \%)$ & 0.150 \\
\hline Type II & $8(15.4 \%)$ & $15(10.1 \%)$ & 0.433 \\
\hline Type III & $13(25.0 \%)$ & $29(19.5 \%)$ & 0.517 \\
\hline \multicolumn{4}{|l|}{ LMCA-LAD angle: } \\
\hline End-diastole [degree] & $161 \pm 12$ & $150 \pm 14$ & $<0.001$ \\
\hline End-systole [degree] & $161 \pm 12$ & $150 \pm 13$ & $<0.001$ \\
\hline \multicolumn{4}{|l|}{ LMCA-LCX angle: } \\
\hline End-diastole [degree] & $154 \pm 12$ & $126 \pm 22$ & $<0.001$ \\
\hline End-systole [degree] & $158 \pm 14$ & $135 \pm 21$ & $<0.001$ \\
\hline \multicolumn{4}{|l|}{ LAD-LCX angle: } \\
\hline End-diastole [degree] & $59 \pm 8$ & $94 \pm 20$ & $<0.001$ \\
\hline End-systole [degree] & $56 \pm 12$ & $83 \pm 21$ & $<0.001$ \\
\hline \multicolumn{4}{|l|}{ Diastolic-systolic range: } \\
\hline LMCA-LAD [degree] & $7.9(0.7-30.4)$ & $7.0(0.5-28.3)$ & 0.742 \\
\hline LMCA-LCX [degree] & $8.3(0.9-22.7)$ & $12.1(0.1-45.8)$ & 0.004 \\
\hline LAD-LCX [degree] & $5.2(0.1-24.6)$ & $12.8(0.1-42.0)$ & $<0.001$ \\
\hline LAD-LCX DSR < $10^{\circ}$ & $42(80.8 \%)$ & $61(40.9 \%)$ & $<0.001$ \\
\hline LAD-LCX DSR $\geq 10^{\circ}$ & $10(19.2 \%)$ & $88(59.1 \%)$ & \\
\hline \multicolumn{4}{|l|}{ Directional angular change: } \\
\hline LMCA-LAD [degree] & $0.4(-19.8-30.4)$ & $-0.9(-28.3-28.4)$ & 0.982 \\
\hline LMCA-CX [degree] & $-5.8(-20.2-22.7)$ & $-10.4(-45.8-34.0)$ & 0.011 \\
\hline LAD-CX [degree] & $2.8(-14.0-24.6)$ & $12.5(-19.9-42.0)$ & $<0.001$ \\
\hline Systolic increase in LMCA-LAD & $26(50.0 \%)$ & $77(51.7 \%)$ & 0.835 \\
\hline Systolic increase in LMCA-CX & $36(69.2 \%)$ & $110(73.8 \%)$ & 0.646 \\
\hline Systolic decrease in LAD-CX & $35(67.3 \%)$ & $123(82.6 \%)$ & 0.035 \\
\hline
\end{tabular}

* LMCA bifurcation types according to LAD and LCX including all LMCA division types. LCX — left circumflex artery; DSR — diastolic- systolic range; LAD — left anterior descending artery; LMCA — left main coronary artery; RCA — right coronary artery 
Table 5. Correlation coefficients for left main coronary artery (LMCA) distal bifurcation angle

\begin{tabular}{lcc}
\hline & $\begin{array}{c}\text { Correlation } \\
\text { coefficients }\end{array}$ & P \\
\hline End-systolic LAD-LCX BA [degree] & 0.826 & $<0.001$ \\
DAC of LAD-LCX [degree] & 0.431 & $<0.001$ \\
DSR of LAD-LCX [degree] & 0.473 & $<0.001$ \\
End-diastolic LMCA-LAD BA [degree] & -0.421 & $<0.001$ \\
End-systolic LMCA-LAD BA [degree] & -0.395 & $<0.001$ \\
End-diastolic LMCA-LCX BA [degree] & -0.773 & $<0.001$ \\
End-systolic LMCA-LCX BA [degree] & -0.661 & $<0.001$ \\
LMCA length [mm] & 0.465 & $<0.001$ \\
CA between aorta and LMCA [degree] & 0.151 & 0.032 \\
LMCA ostial area [mm²] & 0.022 & 0.754 \\
LMCA distal area [mm²] & 0.039 & 0.584 \\
LAD ostial area [mm²] & 0.200 & 0.004 \\
LCX ostial area [mm²] & 0.156 & 0.027 \\
Age [years] & -0.059 & 0.406 \\
Body mass index [kg/m²] & 0.095 & 0.182 \\
Body surface area [m²] & 0.048 & 0.495
\end{tabular}

$\mathrm{BA}$ - bifurcation angle; $\mathrm{CA}$ - cranial angle; DAC — directional angular change; DSR — diastolic to systolic range; LCX — left circumflex artery; LAD — left anterior descending artery

as a result, worse clinical outcomes [5, 27, 30]. Also, in case of pronounced tapering, there is a possibility of underexpansion of distal end of the stent and attempts for obtaining proper expansion with balloon dilatation may further increase the vascular injury. In the present study, LMCA ostial-LMCA distal area and LMCA distal-LAD ostial area ratios were $\geq 1.44$ in 77 (38.4\%) and 96 (47.8\%) patients respectively which implicates moderate to very pronounced tapering structure of LMCA and LMCA bifurcation. It should be remembered that tapering can be more pronounced in the diseased vessels [16].

Tapering of a stenotic vessel is associated with additional challenges such as risk of overexpansion at proximal end if the diameter of the stent is not properly selected or main vessel diameter is higher than the maximal diameter option of the stent. It is recommended to select stent diameter which allows for expansion to the reference diameter of the proximal main vessel, this can be possible with overexpansion of the stent by large balloons [20]. But, overexpansion may lead to stent fracture which carries thrombosis risk [2, 7, 21]. Maximal diameter of most available DESs in the market is $4.0 \mathrm{~mm}$ and the manufactur- ers do not recommend dilating these stents more than $4.5 \mathrm{~mm}$, because of the risk of incomplete sent apposition, plaque prolapse, impaired drug delivery, and strain and dissection of the arterial tissue [9]. In the present study, areas of more than $15.9 \mathrm{~mm}^{2}$ (calculated MLA of diameter $4.5 \mathrm{~cm}$ according to area calculation formula) at LMCA ostial, LMCA distal and LAD ostial regions were found in $63.2 \%, 16.9 \%$ and $3.0 \%$ of patients, respectively.

Cardiac motion alters the LMCA BAs $[11,26]$. It was demonstrated that mean value of LAD-LCX angle decreases and mean value of LMCA-LCX angle increases due to systolic motion [11, 12]. Our findings are consistent with the previous studies. Additionally, we demonstrated that LAD-LCX increases in 49 (24.4\%) patients and LMCA-LCX angle decreases in $55(27.4 \%)$ patients due to systolic motion. These findings are also consistent with recently published study by Handran et al. [14]. Additionally, we found that DSR of LAD-LCX angle was $\geq 10^{\circ}$ in $48.8 \%$ and $<10^{\circ}$ in $51.2 \%$ of the patients.

The European Bifurcation Club has advised routine measurement of LMCA-LAD BA (or distal LMCA BA) for planning bifurcation treatment strategy and for the prediction of procedural outcomes [19, 20, 26, 29]. A T-stent or modified T-stent, or TAP (T-and-protrusion) techniques are considered for T-shaped ( $\geq 70 \%$ ) LMCA distal BA lesions because of its contribution to complete coverage of the bifurcation [19, 20, 26, 29]. On the other hand, culottes or mini-crush techniques are preferred for Y-shaped or V-shaped (< 70\%) distal LMCA BA [19, 20, 26, 29]. Substantive role of distal LMCA BA on procedural side branch (SB) occlusion and patients' outcome is controversial [19, 20, 26]. Previous studies have found that LMCA distal BA $>70^{\circ}$ carries higher risk of SB occlusion than distal $\mathrm{BA}<70^{\circ}$ [1]. COBIS II Registry demonstrated that preprocedural stenosis $\geq 50 \%$ in SB and proximal main vessel, SB lesion length and acute coronary syndrome are independent predictor of increased risk of SB occlusion and $\mathrm{LMCA} P C \mathrm{Pl}$ independent predictor of decreased risk of SB occlusion [13]. In a recently published trial using special risk scoring system to predict SB occlusion found a relationship between distal non-LMCA BA with SB occlusion and no SB occlusion was recorded in LMCA PCI patients. $S B$ occlusion seems to be occurring less frequently in LMCA lesions than in non-LMCA [8]. This may be due to the large structure of SB (LCX) of LMCA lesions as compared to non-LMCA. In the present study, $74.1 \%$ 
of the patients had T-shaped LMCA. Unexpectedly, patients with T-shaped LIMA had larger LAD and CX ostial areas than patients with Y-shaped LMCA, and these areas showed weak positive correlation with distal LMCA angle. These findings might explain why an unfavourable finding of non-LMCA bifurcation is not associated with SB occlusion in LMCA bifurcation lesions. Additionally, in the present study, we found that distal LMCA has moderate positive correlation with DSR of distal LMCA and T-shaped LMCA group has higher DSR of distal LMCA BA. We also found that patients with T-shaped LMCA have longer LMCA than Y-shaped LMCA, but the clinical importance of this finding is not clear.

\section{Limitations of the study}

Major limitation of our study is the inclusion of patients with normal LMCA and patients with diseased LMCA with various degree of stenosis were not included. So, we comment as the distal LMCA angles will not be affected after the stenosis formation at distal LMCA. We can compare normal LMCA with diseased LMCA for investigating the effect of atherosclerosis on these BAs. We did not measure the diameter at the middle of LMCA to better reveal its shape. Additionally, LMCA BAs may be significantly different in heart failure and other structural heart diseases, but patients with structural heart diseases were excluded from the present study.

\section{CONCLUSIONS}

In conclusion, LMCA and LMCA bifurcation have wide variety of dimensions and frequently have tapering shape. Systolic motion significantly affects LMCA-LCX and LAD-LCX angles and mostly results in a reduction of LAD-LCX BA and an increment of LMCA-LCX BA. Patients with T-shaped distal BA was found to have significantly longer LMCA, larger LAD ostial area, larger LCX ostial area and higher DSR of LAD-LCX angle compared to patients with Y-shaped distal BA.

\section{REFERENCES}

1. Aliabadi D, Tilli FV, Bowers TR, et al. Incidence and angiographic predictors of side branch occlusion following high-pressure intracoronary stenting. Am J Cardiol. 1997; 80(8): 994-997, indexed in Pubmed: 9352966.

2. AL-Mangour B, Mongrain R, Yue S. Coronary stents fracture: An engineering approach (review). Materials Sciences and Applications. 2013; 04(10): 606-621, doi: 10.4236/ msa.2013.410075.
3. Athappan G, Patvardhan E, Tuzcu ME, et al. Left main coronary artery stenosis: a meta-analysis of drug-eluting stents versus coronary artery bypass grafting. JACC Cardiovasc Interv. 2013; 6(12): 1219-1230, doi: 10.1016/j. jcin.2013.07.008, indexed in Pubmed: 24355112.

4. Campos CM, van Klaveren D, Farooq V, et al. EXCEL Trial Investigators. Long-term forecasting and comparison of mortality in the Evaluation of the Xience Everolimus Eluting Stent vs. Coronary Artery Bypass Surgery for Effectiveness of Left Main Revascularization (EXCEL) trial: prospective validation of the SYNTAX Score II. Eur Heart J. 2015; 36(20): 1231-1241, doi: 10.1093/eurheartj/ehu518, indexed in Pubmed: 25583761.

5. Chen HY, Hermiller J, Sinha AK, et al. Effects of stent sizing on endothelial and vessel wall stress: potential mechanisms for in-stent restenosis. J Appl Physiol (1985). 2009; 106(5): 1686-1691, doi: 10.1152/japplphysiol.91519.2008, indexed in Pubmed: 19299567.

6. De Caterina AR, Cuculi F, Banning AP. Incidence, predictors and management of left main coronary artery stent restenosis: a comprehensive review in the era of drugeluting stents. Eurolntervention. 2013; 8(11): 1326-1334, doi: 10.4244/EIJV8I11A201, indexed in Pubmed: 23538159.

7. Doi H, Maehara A, Mintz GS, et al. Classification and potential mechanisms of intravascular ultrasound patterns of stent fracture. Am J Cardiol. 2009; 103(6): 818-823, doi: 10.1016/j.amjcard.2008.11.051, indexed in Pubmed: 19268738.

8. Dou K, Zhang $\mathrm{D}, \mathrm{Xu}$ Bo, et al. An angiographic tool for risk prediction of side branch occlusion in coronary bifurcation intervention: The RESOLVE score system (Risk prEdiction of Side branch OccLusion in coronary bifurcation interVEntion). JACC Cardiovasc Interv. 2015; 8(1 Pt A): 39-46, doi: 10.1016/j.jcin.2014.08.011, indexed in Pubmed: 25616815.

9. Foin N, Sen S, Allegria E, et al. Maximal expansion capacity with current DES platforms: A critical factor for stent selection in the treatment of left main bifurcations? Eurolntervention. 2013; 8(11): 1315-1325, doi: 10.4244/ EIJV8I11A200, indexed in Pubmed: 23086760.

10. Fujii K, Carlier SG, Mintz GS, et al. Stent underexpansion and residual reference segment stenosis are related to stent thrombosis after sirolimus-eluting stent implantation: an intravascular ultrasound study. J Am Coll Cardiol. 2005; 45(7): 995-998, doi: 10.1016/j.jacc.2004.12.066, indexed in Pubmed: 15808753.

11. Girasis C, Farooq V, Diletti R, et al. Impact of 3-dimensional bifurcation angle on 5-year outcome of patients after percutaneous coronary intervention for left main coronary artery disease: a substudy of the SYNTAX trial (synergy between percutaneous coronary intervention with taxus and cardiac surgery). JACC Cardiovasc Interv. 2013; 6(12): 1250-1260, doi: 10.1016/j.jcin.2013.08.009, indexed in Pubmed: 24355115.

12. Girasis C, Serruys PW, Onuma Y, et al. 3-Dimensional bifurcation angle analysis in patients with left main disease: A substudy of the SYNTAX trial (SYNergy Between Percutaneous Coronary Intervention with TAXus and Cardiac Surgery). JACC Cardiovasc Interv. 2010; 3(1): 41-48, doi: 10.1016/j.jcin.2009.10.019, indexed in Pubmed: 20129567. 
13. Hahn JY, Chun WJ, Kim JH, et al. Predictors and outcomes of side branch occlusion after main vessel stenting in coronary bifurcation lesions: results from the COBIS II Registry (COronary Blfurcation Stenting). J Am Coll Cardiol. 2013; 62(18): 1654-1659, doi: 10.1016/j.jacc.2013.07.041, indexed in Pubmed: 23954335.

14. Handran $C B$, Garberich RF, Lesser JR, et al. The left main bifurcation angle and changes throughout the cardiac cycle: Quantitative implications for left main bifurcation stenting and stents. J Invasive Cardiol. 2015; 27(9): 401-404, indexed in Pubmed: 25999137.

15. Ho DS, Liu MW, lyer S, et al. Sizing the Gianturco-Roubin coronary flexible coil stent. Cathet Cardiovasc Diagn. 1994; 32(3): 242-248, indexed in Pubmed: 7954772.

16. Javier SP, Mintz GS, Popma JJ, et al. Intravascular ultrasound assessment of the magnitude and mechanism of coronary artery and lumen tapering. Am J Cardiol. 1995; 75(2): 177-180, indexed in Pubmed: 7810498.

17. Kang SJ, Ahn JM, Song H, et al. Comprehensive intravascular ultrasound assessment of stent area and its impact on restenosis and adverse cardiac events in 403 patients with unprotected left main disease. Circ Cardiovasc Interv. 2011; 4(6): 562-569, doi: 10.1161/CIRCINTERVENTIONS.111.964643, indexed in Pubmed: 22045969.

18. Kawasaki $T$, Koga $H$, Serikawa $T$, et al. The bifurcation study using 64 multislice computed tomography. Catheter Cardiovasc Interv. 2009; 73(5): 653-658, doi: 10.1002/ ccd.21916, indexed in Pubmed: 19180660.

19. Lassen JF, Holm NR, Banning A, et al. Percutaneous coronary intervention for coronary bifurcation disease: 11th consensus document from the European Bifurcation Club. Eurolntervention. 2016; 12(1): 38-46, doi: 10.4244/ EIJV12I1A7, indexed in Pubmed: 27173860.

20. Lassen JF, Holm NR, Stankovic G, et al. Percutaneous coronary intervention for coronary bifurcation disease: Consensus from the first 10 years of the European Bifurcation Club meetings. Eurolntervention. 2014; 10(5): 545-560, doi: 10.4244/EIJV1015A97, indexed in Pubmed: 25256198.

21. Marrey RV, Burgermeister R, Grishaber RB, et al. Fatigue and life prediction for cobalt-chromium stents: $A$ fracture mechanics analysis. Biomaterials. 2006; 27(9): 1988-2000, doi: 10.1016/j. biomaterials.2005.10.012, indexed in Pubmed: 16260033.

22. Naganuma T, Chieffo A, Meliga E, et al. Long-term clinical outcomes after percutaneous coronary intervention for ostial/mid-shaft lesions versus distal bifurcation lesions in unprotected left main coronary artery: the DELTA Registry (drug-eluting stent for left main coronary artery disease): a multicenter registry evaluating percutaneous coronary intervention versus coronary artery bypass grafting for left main treatment. JACC Cardiovasc Interv. 2013; 6(12):
1242-1249, doi: 10.1016/j.jcin.2013.08.005, indexed in Pubmed: 24355114.

23. Park SJ, Ahn JM, Foin N, et al. When and how to perform the provisional approach for distal LM stenting. Eurolntervention. 2015; 11(Suppl V): V120-V124, doi: 10.4244/ EIJV11SVA27, indexed in Pubmed: 25983144.

24. Pflederer T, Ludwig J, Ropers D, et al. Measurement of coronary artery bifurcation angles by multidetector computed tomography. Invest Radiol. 2006; 41(11): 793-798, doi: 10.1097/01.rli.0000239318.88270.9f, indexed in Pubmed: 17035869.

25. Ragosta M, Dee S, Sarembock IJ, et al. Prevalence of unfavorable angiographic characteristics for percutaneous intervention in patients with unprotected left main coronary artery disease. Catheter Cardiovasc Interv. 2006; 68(3): 357-362, doi: 10.1002/ccd.20709, indexed in Pubmed: 16892431.

26. Rizik DG, Klassen KJ, Burke RF, et al. Interventional management of unprotected left main coronary artery disease: Patient selection and technique optimization. J Interv Cardiol. 2015; 28(4): 326-338, doi: 10.1111/joic.12211, indexed in Pubmed: 26077351.

27. Russo RJ, Silva PD, Yeager M. Coronary artery overexpansion increases neointimal hyperplasia after stent placement in a porcine model. Heart. 2007; 93(12): 1609-1615, doi: 10.1136/hrt.2006.105981, indexed in Pubmed: 17639098.

28. Sianos G, Morel MA, Kappetein AP, et al. The SYNTAX Score: An angiographic tool grading the complexity of coronary artery disease. Eurolntervention. 2005; 1(2): 219-227, indexed in Pubmed: 19758907.

29. Stankovic G, Lefèvre T, Chieffo A, et al. European Bifurcation Club. Consensus from the 7th European Bifurcation Club meeting. Eurolntervention. 2013; 9(1): 36-45, doi: 10.4244/EIJV9I1A7, indexed in Pubmed: 23552575 .

30. Timmins LH, Meyer CA, Moreno MR, et al. Mechanical modeling of stents deployed in tapered arteries. Ann Biomed Eng. 2008; 36(12): 2042-2050, doi: 10.1007/ s10439-008-9582-0, indexed in Pubmed: 18846425.

31. Windecker S, Kolh P, Alfonso F, et al. Authors/Task Force members. 2014 ESC/EACTS Guidelines on myocardial revascularization: The Task Force on Myocardial Revascularization of the European Society of Cardiology (ESC) and the European Association for Cardio-Thoracic Surgery (EACTS)Developed with the special contribution of the European Association of Percutaneous Cardiovascular Interventions (EAPCI). Eur Heart J. 2014; 35(37): 2541-2619, doi: 10.1093/eurheartj/ehu278, indexed in Pubmed: 25173339. 